Voix et Images

voixetimages

\title{
Le poème dans la poésie, ou les américanités formelles de la poésie
}

\section{Luc Bonenfant}

Volume 34, numéro 1 (100), automne 2008

Pierre Nepveu

URI : https://id.erudit.org/iderudit/019410ar

DOI : https://doi.org/10.7202/019410ar

Aller au sommaire du numéro

Éditeur(s)

Université du Québec à Montréal

ISSN

0318-9201 (imprimé)

1705-933X (numérique)

Découvrir la revue

Citer ce compte rendu

Bonenfant, L. (2008). Compte rendu de [Le poème dans la poésie, ou les américanités formelles de la poésie]. Voix et Images, 34(1), 131-137.

https://doi.org/10.7202/019410ar d'utilisation que vous pouvez consulter en ligne.

https://apropos.erudit.org/fr/usagers/politique-dutilisation/ 


\section{P O ÉS I E}

Le poème dans la poésie, ou les a méricanités

formelles de la poésie

$+++$

LUC BONENFANT

Université du Québec à Montréal

Je commencerai cette chronique en rappelant le mot de Valéry, pour qui "c'est l'exécution du poème qui est le poème. En dehors d'elle, ce sont des fabrications inexplicables, que ces suites de paroles, curieusement assemblées ${ }^{1}$ ». Tout en convenant aisément que la poésie n'est pas qu'une forme (mais Valéry n'écrit pas cela non plus dans son texte...), j'insiste pour dire que la poésie ne peut pas exister sans sa forme. On ne doit pas confondre le langage poétique avec le poème, dont il est toujours une mise en forme. Les recueils que j'ai choisi de vous présenter ont justement en commun cette préoccupation pour la forme - orale chez le premier, métrique chez le second, hybride et populaire chez le troisième - , laquelle soulève par ailleurs plus ou moins explicitement la question de l'américanité du poème.

Slamérica ${ }^{2}$, d'Ivy, est un drôle d'opus, étant dans le même temps un livre et un disque, soit un «livre-disque» (110) selon le mot de l'auteur. Comme s'il devinait par avance notre perplexité quant à la façon d'appréhender son ouvrage, Ivy prend les devants dans son texte de présentation: "Vous n'êtes en rien tenu d'écouter le disque; le livre seul suffit. Rien ne vous force à continuer de lire le livre, le disque est un objet en soi. En d'autres mots, livre et disque peuvent se consommer indépendamment l'un de l'autre [...].» (5) Notez ici le vocabulaire employé, d'autant qu'il fonde toute la poétique de l'ouvrage: nous consommerons plutôt que nous apprécierons Slamérica. Le livre-disque se trouve relégué au statut d'objet: nous sommes, après tout, en terre d'Amérique... Or justement, les vers de cet ouvrage sont, pour le moins, répétitifs:

Dire directement sans salmigon... DIRE

Avant que la mort vienne nous rai... DIRE

1 Paul Valéry, «Leçon inaugurale du cours de poétique du Collège de France», Variété III, IV et $V$, Paris, Gallimard, coll. «Folio essais», 2002 [1944], p. 838. 2 Ivy, Slamérica, Québec/Montréal, Le lézard amoureux/Indica Records, 2008, 114 p. 


\begin{abstract}
Ce que la vie aura voulu nous DIRE
Du tout au tout sans jamais défaillir

DIRE qu'on s'endort en plein après mi... DIRE

Qu'on travaille trop, c'est pas nouveau à DIRE

Qu'on cherche encore un coin de para... DIRE.» (13; Ivy met en majuscules.)
\end{abstract}

C'est sans difficulté qu'on accumulerait les reproches quant aux jeux de mots plus ou moins heureux qui parsèment l'entièreté de l'ouvrage. Disons-le clairement, Slamérica tient mal la route sur le plan esthétique. On se prend même, en cours de lecture et d'écoute, à regretter les subtilités de la poésie sonore contemporaine - je pense ici notamment au fin et audacieux Conte de $F$ de Thomas Braichet ${ }^{3}$.

C'est pour d'autres raisons qu'on aimera Slamérica, car le rythme y constitue une formalisation de l'oralité : la musicalité litanique de «Dire » se situe par exemple entre un certain type de rap et de hip-hop. La forme poétique de l'ouvrage provient de la culture populaire américaine, et c'est probablement pour cette raison que le poète se fait performeur d'un spectacle dont le livre a finalement besoin pour exister. C'est en effet à l'écoute du disque que le livre prend l'ampleur qui doit être la sienne.

Comme toute marchandise, la poésie cherche donc ici à se rendre accessible au plus grand nombre, projetant une certaine idée de la démocratie, politique ou artistique. Ainsi, Slamérica abroge la hiérarchisation des voix. Les épigraphes du livredisque font cohabiter Nietzsche, Paul Celan ou Allen Ginsberg avec Mario Cholette, Jean-Paul Daoust et Led Zeppelin. Le slammeur construit son dialogue intertextuel en fonction d'un principe égalitariste où se trouvent cités des noms dont la résonance n'est plus celle de l'Institution. Un nom en vaut bien un autre parce que c'est sa parole qu'il faut d'abord entendre. Le mot prédomine sur la valeur instituée et Celan peut donc côtoyer Led Zeppelin. L'épigraphe liminaire du livre, «Moi aussi je suis l'Amérique» (9), agit à cet égard comme un véritable programme. Tirée d'un recueil de Langston Hugues, «un des poètes noirs les plus importants des États-Unis - et du monde puisqu'il a directement inspiré le mouvement de la négritude» (9), elle propose de redonner la parole aux exclus. On ne s'étonnera donc pas que la parole amérindienne surgisse çà et là dans les textes, notamment dans "Salsa slam » (54-58), truffé d'extraits du «Discours de 1855» du chef Seattle, dont Ivy nous fournit en note les renseignements pertinents à la compréhension historique des citations. Slamérica est ainsi une œuvre plus politique que poétique, qui séduit à la fois par sa forme orale et sa volonté maintes fois affirmée de redonner une voix aux exclus afin d'ouvrir l'espace de la poésie au plus grand nombre. 
On ne peut pas consommer La vie basse ${ }^{4}$, de Mathieu Croisetière, qu'il faut goûter avec patience et sobriété. Le premier recueil du poète trifluvien se situe à l'opposé du spectre poétique offert par Slamérica.

Ouvrant La vie basse, on est d'abord frappé par le travail métrique qui y règne. De toute évidence, Croisetière connaît les règles élémentaires de la versification, comme en font foi ces poèmes réguliers où il aligne lestement les hexasyllabes. Reste que la part la plus jouissive de son recueil provient peut-être surtout des poèmes où la métrique se fonde sur une spéculation musicale empruntée au jazz (un genre lui aussi américain et populaire, comme le sont le rap et le hip-hop), par exemple dans cette strophe qui intègre le principe du mélange entre rythmes ternaires et binaires alors que la mesure du premier vers se dilue dans celle des deux trisyllabes: «Dans l'hiver blanc de peur/pas d'espace/de répit» (88). Tout au long de son recueil, Mathieu Croisetière évite la monotonie harmonique: tel poème entrecroise hexasyllabes et heptasyllabes alors que tel autre se termine sur un monostique octosyllabique qui coupe la régularité offerte par les distiques hexasyllabiques qui le précèdent.

Assez nombreux dans le recueil, les tétrasyllabes et les heptasyllabes nous rappellent que la poésie est aussi musique. Croisetière parsème d'ailleurs ses poèmes d'ennéasyllabes (un mètre cher à Verlaine comme on sait), ce qui lui permet de repousser l'harmonie rythmique et métrique de la prosodie. L'expérimentation du poète ne cède toutefois jamais à la déroute: la maîtrise du vers est bel et bien là. L'harmonie existe, mais elle doit être attentivement cherchée par le lecteur dont la participation reste essentielle à l'intellection du poème.

Mathieu Croisetière n'est pas qu'un artisan du vers. Le rythme poétique de $L a$ vie basse est tout entier traversé par un timbre particulier, tragique sans être pathétique ou désespéré. Tragique, puisque le poète sait que la fatalité est au rendezvous. Il refuse pourtant d'abdiquer, endossant le vide existentiel pour lui répondre par l'écriture.

La vie basse
la mémoire
est une flaque immobile

Je relis ces visages

qui s'égarent dans les livres (33).

Le poète sait aussi se faire ironique quand il affirme que

La parole nous enfante

sans effort ni aveu

4 Mathieu Croisetière, La vie basse, Trois-Rivières, Éditions d'art Le Sabord, coll. «Recto Verso », 2008, 92 p. 


\begin{abstract}
Mais l'enfant dans l'adulte
recommence à souffrir

Et la rime répète

sa diction ennuyeuse (30).
\end{abstract}

Cette référence narquoise au désœuvrement de la rime découvre une parole bel et bien investie qui évite le piège de la gravité ontologique en retournant le problème de l'affliction existentielle vers l'enjeu formel du poème.

Le sujet poétique, chez Croisetière, semble avoir intégré la leçon d'Icare; il met en place des stratégies rhétoriques qui lui permettent d'éviter la vanité d'une suffisance qui serait autrement pathétique, voire lénifiante. Ainsi, la mort, thème présent depuis le tout premier poème, apparaît en définitive comme un lieu de scintillement alors que, dans le dernier poème, sa fatalité est embrassée par le sujet qui trouve une «joie» dans le fait de "partir/dans l'audace de mourir» (92). Il vogue de ce fait vers l'extase cosmique, dans «l'espacement des étoiles» (92), rappelant que la condition tragique de l'homme ne doit pas être pensée dans la seule exiguïté de l'espace terrestre. Dans La vie basse, le poème et la poésie, ou pour reprendre une vieille dichotomie, la forme et le fond, engendrent le plein déploiement d'une parole qui ne peut se réduire à sa seule plainte ontologique.

Il s'est passé presque dix ans entre la parution du premier recueil de Patrick Lafontaine, L'ambition du vide, pour lequel il avait remporté le prix Émile-Nelligan, et celle d'Au lieu de l'abandon/Mes êtres ${ }^{5}$. Étant donnée la qualité de ces deux recueils, on ne peut que se réjouir de n'avoir eu à attendre que deux ans pour que Lafontaine nous livre son troisième recueil, Homa Sweet Home ${ }^{6}$.

Là où Slamérica raconte une expérience urbaine générique où Chicago, New York ou Montréal sont interchangeables, Homa Sweet Home livre un portrait sans complaisance d'un quartier populaire de Montréal, quartier au demeurant souvent violent parce que défavorisé. Commençons cependant par le début, c'est-à-dire par ce titre riche et ambigu puisqu'il "n'y a pas de titre sans lisibilité d'une trace», comme l'écrit Derrida qui ajoute qu'il «n'y aura titre qu'au moment où la possibilité d'une lecture se sera fixée ${ }^{7}$ ».

D'abord, Homa Sweet Home est un titre volontiers ironique puisque la douceur supposée du foyer est ici celle d'un «trou [où] les uns se piquent s'entretuent s'enlarvent [et] les autres sucent des bites s'ouvrent les veines déboulent les escaliers » (40). Le poète ne compte pas nous préserver de cette violence dont la vie,

5 Patrick Lafontaine, L'ambition du vide, Saint-Hippolyte, Éditions du Noroît, 1997, 66 p. ; Au lieu de l'abandon/Mes êtres, Montréal, Éditions du Noroît, 2006, 158 p. 6 Patrick Lafontaine, Homa Sweet Home, Montréal, Éditions du Noroît, 2008, 96 p. 7 Jacques Derrida, "Titre à préciser», Parages, Paris, Éditions Galilée, coll. «La philosophie en effet», 1986, p. 225 et p. 226. 
dans «Homa», ne fait finalement jamais l'économie. Mais le quartier Hochelaga de Patrick Lafontaine n'est pas celui immortalisé par le film de Michel Jetté ${ }^{8}$. Exit le caractère spectaculaire de la violence de la guerre des motards; c'est plutôt celle, ordinaire et quotidienne, des résidants du quartier qui retient le poète: «à 15 ans Hochelaga montre ses seins durs et blancs ses bottes de manga cirées rose lance ses hanches dans tous les yeux brûle la fin d'un joint offre sa liberté» (19). Le sexe et la drogue sont d'ailleurs partout présents dans ce recueil, de même que le personnage de la prostituée, jamais rabaissée, souvent même décrite avec tendresse.

Ensuite, le titre dépasse le strict jeu de mots sur un syntagme figé alors que la langue dans laquelle il est rédigé rappelle aussi que des Irlandais ont jadis vécu dans «Homa», plus précisément dans la partie Maisonneuve du quartier qui était alors considérée comme la Pittsburgh du Canada. On chercherait toutefois en vain des références sociologiques dans Homa Sweet Home, qui n'est heureusement pas un recueil historique. Reste que le titre évoque implicitement le lien historique qui existe entre les communautés francophone et anglophone du quartier, toutes deux ouvrières. Et plutôt qu'à leur langue (surtout que la communauté anglophone est aujourd'hui largement francisée), le recueil s'arrête justement à cette condition ouvrière partagée ou, plus précisément à la violence et à l'aliénation qui lui sont conséquentes et auxquelles le poète espère échapper quand il écrit que «depuis que le fou d'en face a disparu, [il a] peur de sa place» (87).

Enfin, Homa Sweet Home, par la contraction initiale de son premier mot, nous dit aussi que le quartier du poète n'est plus aujourd'hui seulement celui des ouvriers et des défavorisés. Logent en effet à leurs côtés de jeunes professionnels, sorte de mascarets sociaux qui bouleversent la donne de ce lieu où «la mort n'est plus l'énigme.//C'est aujourd'hui la jeunesse» (62). Est-ce que ce sera pour le meilleur ou pour le pire?

\author{
au dessus d'Ontario \\ parmi les réverbères \\ les phares d'autos \\ sur un feu rouge \\ passe la lune \\ les enfants rêvent \\ allongés de s'en sortir (58).
}

L'espoir est faible, mais Lafontaine refuse de prendre la posture moraliste du contempteur. Le moyen d'éviter le renversement provoqué par cette marée sociale semble pour lui résider dans l'appel véritable d'une communion dont son recueil se fait finalement le témoin le plus sensible.

En effet, la médiation du poème conduit à la possibilité d'une cohabitation éventuelle qui ne serait pas possible sans la tonalité lyrique particulière qui traverse

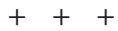

8 Michel Jetté, Hochelaga, Saint-Laurent, Films Lions Gate, 2000. 
le recueil, sans cette capacité réelle du sujet à se dégager de lui-même pour laisser surgir la voix des autres: «j'ai tellement écrit je que je me donne/mal au cœur» (14). Le poète fournira aux exclus et aux dépossédés les mots dont ils ont besoin pour s'exprimer. Son Je n'est désormais plus le sien quand il écrit:

\author{
je parque ma Nissan verte \\ la portière frotte \\ le trottoir j'ai vendu \\ mes disques pour un poulet \\ rôti [...] (17).
}

Le sujet lyrique de Lafontaine est d'abord et avant tout un sujet poreux dont la transparence laisse place à une altérité généreuse. Se perdre dans ceux qu'il fait exister, voilà un des enjeux de cette voix dont l'intelligence réside dans l'affection réelle que le poète ressent à l'égard de ceux avec qui il cohabite, au «1646/rue Joliette» (14). Même s'il pense être réduit à une présence fantomatique, seul la nuit devant son ordinateur «muet, transparent et froid» (23), malgré ses récriminations et ses suppliques quand il hurle son abattement - «plus je t'écris plus tu m'écœures Hochelaga-l'avide tu n'aimes personne en avalant tout le monde» (79) -, le poète n'abandonnera pas et son jeu en aura valu la chandelle.

La puissance de son projet est d'ailleurs tout autant scripturale qu'artistique. Sur le plan de l'écriture, Patrick Lafontaine offre une trame qui mélange habilement poèmes en vers et poèmes en prose pour ainsi servir une forme populaire du discours poétique. Quand ils sont en vers, les poèmes privilégient une syntaxe parlée dont la réussite repose sur de nombreux enjambements. Les poèmes en prose, eux, évitent souvent toute ponctuation pour pencher vers la prolixité d'un discours qui repose sur l'urgence de son dire $^{9}$. «Pour oublier mon silence je suis venu où tout le monde parle» (70), nous rappelle le poète qui emprunte aux habitants d'Hochelaga leur phrasé pour le mettre en vers ou en prose: là réside un des aspects de la communion profonde offerte par son recueil.

Sur le plan pictural, dix-neuf photos illustrent Homa Sweet Home. Toutes prises par l'auteur, elles disent chaque fois l'éclat possible de ce quartier grâce à la vivacité des couleurs ou aux nuances subtiles des tons qui s'y agencent. Les clichés de la minoterie ADM ou de la raffinerie Lanctic, sises rue Notre-Dame, rappellent le passé industriel encore omniprésent du quartier alors que ceux de l'église Très-SaintRédempteur, rue Adam, témoignent de la somptuosité de certains de ses édifices. Le photographe embrasse aussi l'ordinaire de son quartier. Les images léchées qu'il offre d'un escalier en colimaçon, d'une corniche ou d'une ruelle et de ses balcons offrent une perspective nouvelle sur Hochelaga. Hors de tout doute, avec ce troisième recueil, Patrick Lafontaine a composé une ode sensible et intelligente à un quartier de Montréal dont on ignore trop souvent la beauté.

9 Certains poèmes en prose du recueil sont cependant composés de phrases courtes qui semblent autant de constats lapidaires n'admettant aucune forme de procès. Encore ici, la syntaxe reste "populaire», presque rudimentaire, en tous les cas réduite à sa plus simple expression. 
À l'évidence, Slamérica n'a pas la même valeur esthétique que La vie basse et Homa Sweet Home. Mais on ne saurait être indifférent à aucun de ces recueils qui, tout en proposant des expériences fort différentes de la poésie, travaillent chacun à leur façon les formes. Le poème y est chaque fois une préoccupation réelle, un enjeu où l'architectonique s'adjoint au formel du langage alors que le slam du premier, le rythme jazzé du second et la syntaxe urbaine et populaire du troisième inscrivent l'expérience poétique en terre d'Amérique. 